\title{
Foraging on the radial-arm maze: Effects of altering the reward at a target location
}

\author{
JOHN D. BATSON \\ Furman University, Greenville, South Carolina \\ MICHAEL R. BEST \\ Southern Methodist University, Dallas, Texas \\ DEBORAH L. PHILLIPS \\ Furman University, Greenville, South Carolina \\ HEMLATA PATEL \\ Southern Methodist University, Dallas, Texas \\ and \\ KEVIN R. GILLELAND \\ Furman University, Greenville, South Carolina
}

\begin{abstract}
Thirsty rats were trained to collect small water rewards from the end of each arm of an eightarm radial maze. During these training trials and subsequent testing trials, the subjects were allowed to choose a maximum of eight arms. "Preference" for a target maze location was studied by noting when, in the sequence of eight choices, the target was selected. During testing, when one maze location was consistently devoid of water, rats decreased their preference for this arm over trials (Experiment 1). Similarly, rats that learned a saccharin-lithium association demonstrated lower preferences for a maze location that consistently held the conditioned saccharin solution. This was true for animals that received saccharin-lithium conditioning on the maze (Experiment 3A) and for animals conditioned to saccharin in a separate context (Experiment 3B). An increase in preference for a target maze location consistently containing a sweet chocolate milk solution was observed in animals that were water- and food-deprived (Experiment 2). These studies demonstrate that animals will modify their responses toward (preferences for) maze locations that predictably contain an altered reward.
\end{abstract}

The radial-arm maze has become a popular and important apparatus in the study of rat behavior in recent years. This maze has a central "hub" and a number of arms or alleys radiating away from the central area. Typically, food or liquid rewards are situated at the end of each maze arm and the hungry or thirsty rat is then allowed to collect the rewards. Rats learn quickly to select a large number of different arms before repeating an already visited location. For example, on a 17-arm maze they can select about 15 different arms before repeating a previous choice. Moreover, rats often appear to select maze arms in a rather haphazard fashion instead of selecting arms sequentially (Olton, 1978), although animals some-

The studies reported here were supported by Grant MH40976 from the National Institute of Mental Health to the first author and Furman University's Committee on Research and Professional Growth. Deborah L. Phillips was a Dana Undergraduate Research Fellow at Furman University during the time these studies were conducted. We thank Michael Domjan for allowing us to use his radial-arm maze.

Reprint requests should be sent to John D. Batson, Psychology Department, Furman University, Greenville, SC 29613 times use sequential or algorithmic response patterns (Einon, 1980; Foreman, 1985).

Radial-arm maze studies verify that foraging rats usually develop very efficient strategies. Three examples of such efficient behavior include: (1) rats' selection of almost all of the different arms before returning to a location where the bait has been depleted (Olton \& Samuelson, 1976); (2) the fact that when running on a maze where one arm either is consistently empty or contains a taste that has become aversive through conditioning, rats decrease their visits to that arm relative to alternative arms (Melcer \& Timberlake, 1985); and (3) the fact that when running on mazes that have central arenas of different sizes, rats adopt strategies that decrease the time/energy required to gather the rewards (Yoerg \& Kamil, 1982).

Although it is clear that rats perform radial-arm maze tasks efficiently in many situations, a number of other manipulations that should elicit efficient responding have yet to be explored. In each of the experiments described here, thirsty subjects first were observed during a baseline condition, in which small water rewards were located at the end of each maze arm. Because the depleted baits 
were not replaced, the animals learned to avoid locations visited earlier in each day's trial. Following baseline, the reward located on one of the arms of the maze was altered in such a way that that location should have been visited more or less often over successive trials. Together, these studies describe how foraging rats respond to changes in the quality of edibles located in specific locations.

\section{EXPERIMEN' 1}

A simple way to modify the value of a foraging site is to remove the reward previously located there. Numerous T-maze studies have shown that animals extinguish their responses toward a maze arm that no longer contains a reward. On the eight-arm maze, animals accustomed to drinking water in one location should decrease their willingness to traverse this target maze arm once the reward is removed. In this study, two groups of thirsty rats first learned to sample maze locations for small water rewards at the end of each arm. After this initial baseline phase, the experimental rats continued to find water on all arms except the one site where the reward was consistently absent. The control group of animals was treated similarly, except that the empty arm of the maze was determined randomly each day.

\section{Method}

Subjects. The subjects were 12 male rats derived from LongEvans rats (Blue Spruce Farms, NY) born and reared in the animal facilities at Furman University. They were approximately 15 weeks old at the beginning of the study and were experimentally naive. They were maintained individually in standard stainless steel cages $(24 \times 24 \times 30 \mathrm{~cm})$ in a Wahmann rack and always had food (Purina Rat Chow) available ad lib in the home cages. Access to water was restricted to $30 \mathrm{~min}$ per day approximately 10 days before the experiment. This water-deprivation schedule remained in effect for the duration of the study. Lights were automatically controlled and were on between 0700 and $2100 \mathrm{~h}$. Experimental sessions were conducted between 1200 and $1400 \mathrm{~h}$, and the 30-min home-cage water session occurred immediately after each experimental session.

Apparatus. The testing apparatus was a radial-arm maze like that described by Olton and Samuelson (1976). The arms were $86.5 \mathrm{~cm}$ long and $7.5 \mathrm{~cm}$ wide. Each arm radiated away from one side of the central octagonal platform, which measured $33 \mathrm{~cm}$ in diameter. The entire maze was constructed of wood and was situated $75 \mathrm{~cm}$ above the floor on a standard laboratory stool. Each arm was assigned a number from 1 to 8 (but was not physically marked) and was always situated in exactly the same orientation within the test room. This test room, located approximately $20 \mathrm{~m}$ from the main animal room, contained various types of lab equipment, including a table, machines, a lab rack, and so on. A ventilating unit located above and to one side of the maze emitted a constant noise. Bottle caps (with plastic liners) were fixed to the ends of the maze arms, and these served as liquid containers when the animals were running the maze. When the maze arms were baited, $.2 \mathrm{ml}$ of water was placed in each bottle cap before the animal was placed on the central platform. Once the animal drank the liquid on a given arm, that arm remained empty for the duration of the animal's time on the maze that day. It is important to note that there were no visual or other cues to signal the presence or absence of liquids in the bottle caps: the animals had to traverse the length of each arm before discovering whether the caps held anything. Moreover, ventilation in the testing room circulated air constantly, thereby ensur- ing that odor cues were evenly distributed throughout the room and could not be used to locate specific baits.

Procedure. The experiment consisted of three phases. The exploration phase lasted for 7 consecutive days, during which all animals were treated alike. Each animal was removed from its home cage, transported to the nearby testing room, and allowed to explore the eight-arm maze. During each of these daily "trials," the animal remained on the maze for $10 \mathrm{~min}$ or until it had consumed the $.2 \mathrm{ml}$ water reward located at the end of each arm, whichever came first. By the end of this phase, all animals were actively sampling the maze arms and consuming the liquid rewards.

The baseline phase of the experiment followed; this was similar to the exploration phase and was designed to provide a standard measure of each animal's performance before the groups were administered separate treatments. During this phase and for the rest of the study, the time to complete each trial was recorded, as was the order and number of different arms chosen. Moreover, from this point on, the animals were allowed to select a total of only eight arms on the maze during each daily trial. To perform optimally in this situation, thirsty animals should, of course, select eight different arms. An arm choice was recorded when the rat arrived within $5 \mathrm{~cm}$ of the end of an arm. (In our lab, animals that have had a few days experience on the maze rarely walk only part way down an arm. When animals leave the central platform-by placing all four feet on an arm-they virtually always walk all the way to the end of the arm.) This baseline phase consisted of eight trials administered over a period of 9 days.

The testing phase of the study followed and consisted of 12 trials conducted over a period of 13 days. Each trial consisted of eight choices. Prior to the first testing trial, the animals were assigned randomly to one of two groups. During each testing trial, water was available on seven of the maze arms and the remaining arm was left unbaited. The only difference between groups was the location of the empty arm. For the experimental group $(n=8)$, it was always Arm 7. This target arm was chosen more or less randomly, the only restriction being that all animals tended to choose the target during baseline and both groups tended to choose the target in apparently random fashion (i.e., not consistently early or late in each trial). For the control group $(n=4)$, the target was determined randomly from day to day with the following restrictions: a given maze arm could not be vacant on 2 successive days and, within a block of eight successive trials, each maze arm had to be vacant once.

Statistical analyses. Data were initially analyzed using mixed analyses of variance-of the type labeled "SPF-p . q" by Kirk (1968)-to reveal effects between subjects (treatment conditions), within subjects (over days or trials), and of interactions. Subsequent analyses utilized within-subjects $t$ tests.

\section{Results and Discussion}

In this study, our primary interest was in what we call "preference" for the target arm. Preference was computed on the basis of when a particular maze arm was chosen within a given trial. If the specified arm was the first arm chosen, a score of 8 (the maximum) was recorded; if it was chosen second, the score would be 7, and so on. Thus, arm-preference scores could range from 8 to 0 (if the arm was not chosen in that trial). If only random factors determine selection of a given arm and if an animal chooses $\mathbf{8}$ different arms, the preference score expected by chance alone equals 4.5 .

Preferences for the target arm (No. 7) during baseline and testing phases are shown in Figure 1 in successive two-trial blocks. During baseline, both groups selected eight arms per trial and all arms contained water. The 


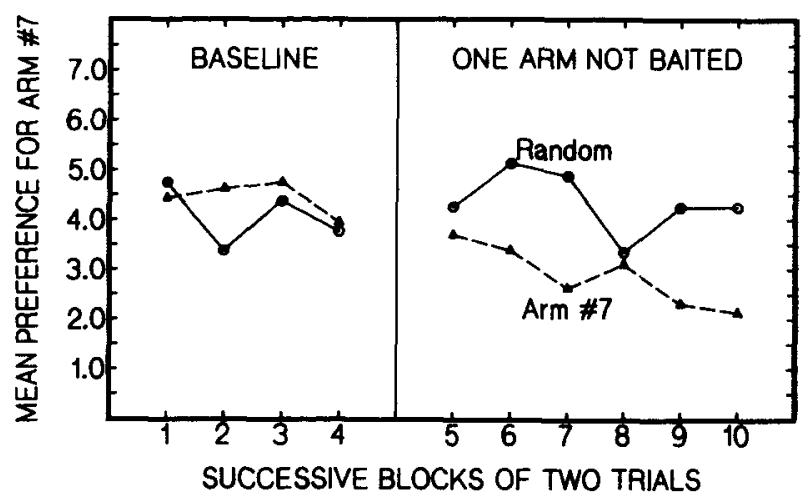

Figure 1. Mean preferences for the target arm during Experiment 1 . Both groups were allowed eight arm choices during each of eight baseline trials with a water reward located on each arm of the maze. During the final 12 trials, one of the maze arms was not baited. This empty arm was consistently Arm 7 for the experimental group and was randomly determined over trials for the control group.

differences in preference for the target arm were nonsignificant. Moreover, the two groups did not differ significantly in number of different arms selected or in total time taken to complete eight choices per trial during baseline [all $F$ s $(1,10)<1.0$ ].

During testing trials, however, the experimental group demonstrated a steady decrease in preference for the target arm. The difference between groups was significant during testing $[F(1,10)=9.00, p<.05]$. These group differences were due primarily to the decrease in target preference in the experimental animals, for this group preferred the target less at the end of testing (last two blocks of trials combined) than during baseline (first four blocks of trials on left in graph) $[t(7)=4.62, p<.05]$. The control group showed no significant difference in target preference in an analogous comparison $[t(3)=.20$, n.s.]. The groups did not differ significantly during testing in number of different arms selected or in the total time to complete trials $\left[F_{\mathrm{S}}(1,10) \leq 1.30\right]$.

In a related experiment, Melcer and Timberlake (1985) allowed rats to sample repeatedly from four maze arms, three of which always contained water and one of which was always empty. They observed a similar decrease in "preference" for (actually, a decrease in percentage of visits to) the empty arm of the maze. Their finding and ours confirm that rats foraging on a maze will alter their performance in relatively efficient ways when a given location holds no reward.

\section{EXPERIMENT 2}

Animals in Experiment 1 decreased their tendency to visit a maze location when that spot no longer held a water reward. This outcome is analogous to that of T-maze studies that show extinction of running to a previously rewarded location. Other maze studies have documented that more vigorous responding occurs when rats are shifted from one reward to a larger or more concentrated sweet reward (e.g., see Flaherty, 1982, for a review of positive contrast effects). The purpose of Experiment 2 was to determine if our measure of arm preference might detect an increase in the tendency to visit a target when visits to that site were rewarded with a highly preferred substance. After a baseline phase, in which two groups learned to select maze locations that contained only water, a testing phase was begun with water on seven of the arms and a sweet chocolate milk solution on one arm. This target site was consistent over days for one group and randomly determined daily for the control group. Because chocolate milk is typically considered to be an especially effective reinforcer, we expected thirsty animals that found the special bait in the same site over days to develop increased preferences for this location. Later in the study, the animals were food deprived to determine if deprivation condition influenced preference for the target arm.

\section{Method}

Subjects. Sixteen animals, like those described in the previous experiment, were used in this study.

Procedure. This study was like Experiment 1 in all unspecified respects. Following an exploration phase and eight baseline trials, the subjects were assigned to one of two groups ( $n=8$ in each). During all subsequent trials, water was placed on seven of the maze arms on each trial and .5 $\mathrm{ml}$ of a sweet chocolate solution $(10 \%$ Hershey's chocolate syrup $+50 \%$ Eagle brand condensed milk + $10 \%$ sucrose $+30 \%$ tap water, all percentages by weight) was placed on the remaining arm. The location of the chocolate solution was always Arm 6 for the experimental group, but was determined randomly (according to the randomization procedure noted in the previous experiment) for the control group. The target arm for the experimental group (Arm 6) was chosen more or less randomly as described in Experiment 1.

The two water-deprived groups were tested for eight trials, which were conducted over a period of 9 days. During this time, we expected the two groups to perform differently on the maze because the sweet reward was consistently found on Arm 6 for only the experimental animals. However, no differences emerged here and both groups continued to perform as they had during the earlier baseline trials. Therefore, a second testing phase was begun that was identical to the first with the single exception that both water and food were restricted in the home cages for both groups. Food deprivation was accomplished by allowing the animals to eat and drink freely for $60 \mathrm{~min}$ between 1400 and $1600 \mathrm{~h}$ daily. After 4 such deprivation days, the animals were given a series of 8 trials (over successive days) on the maze again. Water and the chocolate solution were available on these trials as described for the initial testing phase.

\section{Results and Discussion}

During the time the two groups were water deprived, their mean preferences for the target arm remained equivalent during baseline trials. Moreover, the groups remained similar even after the chocolate solution was first placed on one arm of the maze. These preference scores are plotted in the left portion of Figure 2. Neither the main effects (groups, trials) nor the interaction were statistically significant during this time $(F \mathrm{~s} \leq 1.76)$, Moreover, the two groups did not differ significantly in number of different arms selected or in total time to complete the trials while they were water-deprived $(F \mathrm{~s}<1.0)$. 


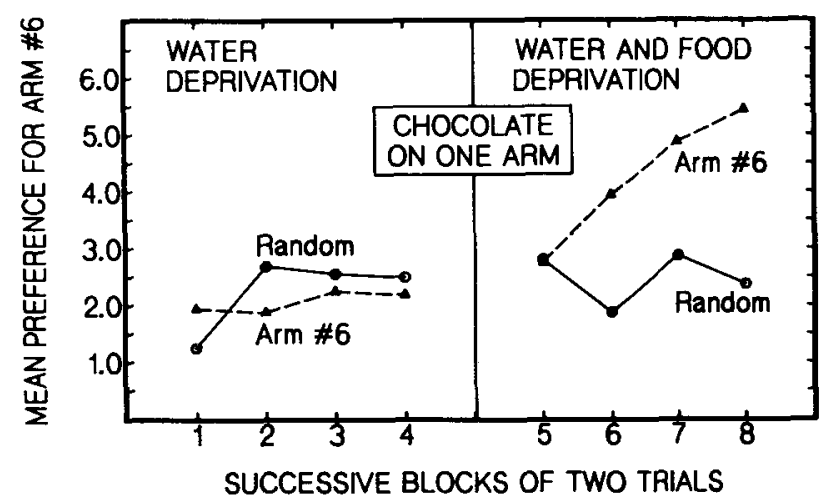

Figure 2. Mean preferences for the target arm during Experiment 2. Following a series of training trials in which water was located on all maze arms, a chocolate milk solution was placed on one arm. The location of the chocolate was consistently Arm 6 for the experimental animals and was determined randomly for control animals. Preferences are plotted for the target arm during water deprivation on the left and during subsequent food and water deprivation on the right.

However, during subsequent food- and waterdeprivation trials, preference for the target arm increased in the experimental group, although it remained relatively stable in the control group. These outcomes are plotted in the right half of Figure 2 . The difference between groups over trials was significant $[F(1,14)=6.49$, $p<.05]$, indicating once again that foraging behavior is modified according to the predictability of finding certain types or qualities of food in specific locations. The effects of repeated testing days and the interaction of groups $x$ days were both significant during the final (water and food deprivation) testing phase $[F \mathrm{~s}(7,98)=$ 3.54 and 3.00 , respectively, $p s<.05]$. The difference between groups here is due primarily to the increase in target preference in the experimental animals; these rats showed a relative increase in this measure between the time they were water deprived (left-hand portion of graph) and the end of testing during the food-deprived sessions (final two blocks of trials) $[t(7)=3.85, p<.01]$. The analogous comparison for the control group was nonsignificant $[t(7)=.80$, n.s. $]$. Finally, the groups were not significantly different in number of different arms chosen across food-deprivation trials and in time to complete these trials $[F \mathrm{~s}(1,14) \leq 4.06]$.

The animals in this experiment developed a heightened preference for a maze location that reliably contained a bait that was sweet tasting and high in caloric value. This outcome is similar to the results of several studies by Bolles and his colleagues (Bolles, Hayward, \& Crandall, 1981; Mehiel \& Bolles, 1984). Subjects in those studies were given experiences with two separately flavored liquids. Each liquid also differed in caloric value. Hungry rats developed preferences for the flavor cue that was associated with the higher calorie liquid. Animals in the present study developed preferences for the spatial location of the high-calorie bait. Thus, deprived animals can learn to identify such rewards by remembering appropriate environmental information as well as relevant taste cues.

It is especially interesting that preference for the target arm depended upon deprivation condition. Hunger, but not thirst, motivated the animals to change their preference for the chocolate liquid location. The gradual increase in target preference during food-deprivation suggests that the experimental animals had not learned much about the chocolate location during the earlier waterdeprivation period. A similar finding was noted by Spence and Lippitt (1946), who used a Y-maze with thirsty and hungry rats.

\section{EXPERIMENT 3A}

Animals foraging in the natural environment must occasionally experience flavors that are toxic. One consequence of ingesting such an item should be avoidance of that flavor in the future. Another possible result is future avoidance of the location containing that food. Laboratory studies have clearly shown that environmental cues are conditionable by illness, at least in some situations (Batson \& Best, 1979; P. J. Best, M. R. Best, \& Henggeler, 1977). From an ecological perspective, the result of an illness episode during foraging should thus be twofold. First, animals should avoid consuming the flavor associated with illness in the future no matter where it is encountered. Second, animals should learn to avoid the location that reliably contains the aversive flavor, since visits here would represent less than optimal foraging behavior.

Melcer and Timberlake (1985) poisoned rats that consumed a saccharin solution in one location of a four-arm maze. As expected, subjects in that experiment subsequently made fewer visits to the saccharin arm. Experiments $3 \mathrm{~A}$ and $3 \mathrm{~B}$ in the present report extend the analysis of this flavor conditioning effect. In our studies, a conditioned aversion was established to a distinctive saccharin solution. The willingness of animals to visit a target maze location containing this solution was then observed during the testing phase. In Experiment 3A, saccharin conditioning was conducted on the maze. Animals that had learned to forage the maze for water rewards subsequently encountered a distinctive saccharin solution on one target arm. Experimental animals that selected the saccharin location were then injected with lithium to induce illness. Control animals were not injected. Our prediction was that the experimental subjects would decrease their visits to the target location as well as develop an aversion for the saccharin fluid.

\section{Method}

Subjects and Apparatus. Eight subjects were used in this experiment. They were similar to, and were maintained in the same way as, subjects in Experiments 1 and 2. All materials and apparatus used were identical to those used in previous experiments.

Procedure. The procedures used here were identical to those noted in Experiments 1 and 2 in all unspecified respects. Follow- 
ing an exploration phase, the subjects were administered six baseline trials, which were conducted over successive days.

The testing phase began the following day and consisted of one trial per day for $\mathbf{8}$ successive days. Prior to the first of these trials, the animals were assigned randomly to either the experimental or the control group ( $n=4$ in each). During these testing trials, water was available on seven of the maze arms and a saccharin solution $(.2 \mathrm{ml} ; .15 \%$ sodium saccharin, $w / v$ in tap water) was placed on Arm 1. This target arm was selected more or less randomly, as described in Experiment 1. The experimental animals were injected with lithium (.15 M, $1.5 \mathrm{ml}$, ip) after completing each trial if they had chosen the saccharin arm on that trial. The controls were never injected. On the initial test trial, all subjects in both groups selected the target arm and drank the saccharin located there. The temporal delay between the time Arm 1 was chosen and the time the experimental subjects were injected with lithium was approximately 1-3 min, depending upon when Arm 1 was chosen in the eightarm sequence. Water was made available in the home cage for $30 \mathrm{~min}$ approximately $3 \mathrm{~h}$ following test trials.

These procedures were followed on each of the eight test trials with the single exception that lithium was not administered to experimental subjects on trials when the saccharin arm was not visited.

\section{Results and Discussion}

The two groups differed very little during the baseline phase when treatments were similar. Preferences for the target arm, Arm 1, were roughly equivalent, as were total number of different arms selected and time to complete eight choices per trial (all $F \mathrm{~s}<1.00$ ). The two groups' baseline preferences for the target arm and number of different arms visited per trial are shown in the left portions of Figures 3 and 4, respectively.

During the testing phase of the experiment, subjects in the two groups differed in their willingness to select the target saccharin arm. These differences between groups were significant $[F(1,6)=21.03, p<.01]$ and are plotted in the right portion of Figure 3. Nonsignificant differences were noted for the effects of repeated trials and the trials $\times$ groups interaction $[F \mathrm{~s}(7,42)=.63$ and 1.40 , respectively]. Subjects in the experimental group steadily decreased their preferences for the saccharin location, whereas the control subjects maintained a relatively higher

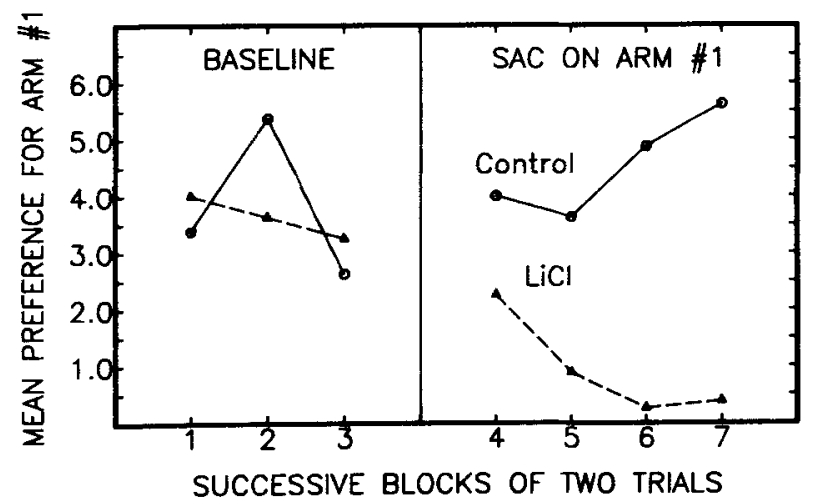

Figure 3. Mean preferences for the target arm during Experiment 3A. During baseline, both groups found water on all maze arms. Saccharin was present on the target arm during the final eight trials, and animals were either injected with lithium if they selected the target during each of these trials ( $\mathrm{LiCl}$ group) or were not injected (control group).

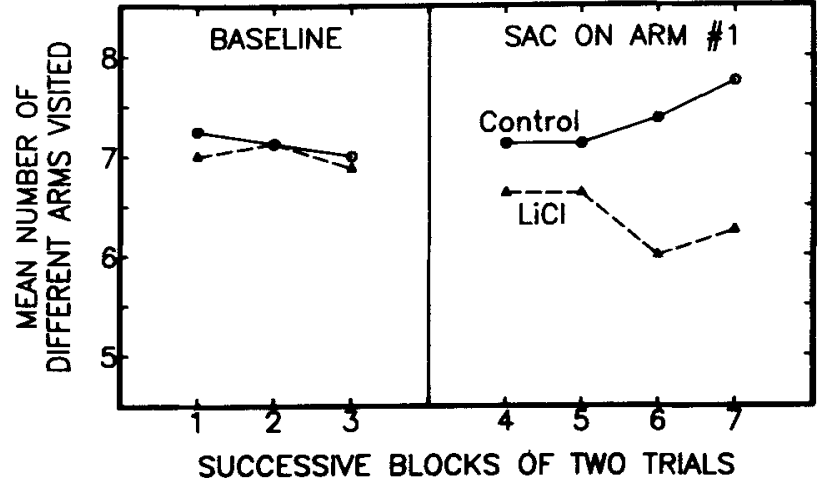

Figure 4. Mean number of different maze arms selected during Experiment 3A. Because the animals were required to make eight choices during all trials, the maximum possible score was 8.0. Injection patterns are described in Figure 3.

preference for the same arm. The graph might suggest that part of this between-groups difference arises from an increase in target preference in the control group, and the results of Experiment 2 (in which rats increased their preferences for a highly palatable solution) might also predict such an outcome. However, most of the betweengroups difference here is due to the decrease in target preference in the experimental group. Within-subjects $t$ tests were computed by comparing overall baseline preference scores (mean of the first three blocks of trials) with preference scores at the end of the experiment (mean of the last two blocks of trials). These tests revealed a significant decrease in preference for the target arm among the experimental animals $[t(3)=5.13, p<.02]$, but no significant difference for the control group $[t(3)=1.80$, n.s.]. Notice that mean preference for the target location was nearly zero-indicating that most animals completely avoided this location-for the last two blocks of trials. Thus, the difference between the two groups during the time saccharin was on the maze is primarily due to the decreased willingness of the experimental animals to choose the target arm.

Because the experimental animals occasionally avoided the saccharin arm of the maze but were still required to select a total of eight arms on each testing trial, these subjects visited a smaller number of different arms than control animals. This difference, which is shown in the right portion of Figure 4, was significant $[F(1,6)=7.30$, $p<.05]$. During testing, there were also significant effects of days and the groups $\times$ days interaction $[F \mathrm{~s}(7,42)$ $=2.82$ and 2.43 , respectively, $p \mathrm{~s}<.05$ ]. The mean number of different arms visited over the final two blocks of testing was significantly different from baseline performance only for the experimental animals $[t(3)=6.97$, $p<.05]$, not for the controls $[t(3)=1.26$, n.s.].

It is also worth noting that the lithium injections administered to the experimental animals may have produced effects other than the conditioning of an avoidance of the saccharin fluid and the saccharin location. Compared either with the control subjects or with their own baseline, experimental animals engaged in more "rearing" 
and "sniffing" responses following several conditioning trials, and they defecated and urinated more as well. These response patterns were accompanied, in two subjects, by a sporadic refusal to drink even the water on nontarget arms of the maze. This may suggest that lithium served to condition aversions not only to the saccharin and the saccharin location but also perhaps to water or to the entire maze environment. However, in this experiment, any evidence of this type of conditioning did not appear until well after the experimental animals :vere obviously avoiding both drinking the saccharin bait and approaching the saccharin location. It is also important to remember that the two groups did not differ in their preferences for the seven nontarget arms during testing [largest $F(1,6)=$ 1.16, n.s.]. Finally, because only the experimental group received lithium injections, one might expect the experimental animals to differ from controls in time taken to complete the eight choices in each testing trial. In fact, there was no such statistical difference $(F<1.00)$.

The results of Experiment $3 \mathrm{~A}$ indicate that when rats drink a novel taste in a specific location on an eight-arm radial maze and are then made ill, preference for the arm on which this taste is located, as well as preference for the taste, decreases. This type of procedure models the situation that might occur for animals foraging in a natural environment. A toxic and novel food is encountered in one of several foraging locations and illness ensues. Subsequently, the animals are less likely to forage in the tainted location. Experiment 3B investigated a different situation. Would animals decrease their preference for a foraging location if they encountered a food there that was made aversive in another context?

\section{EXPERIMENT 3B}

\section{Method}

Subjects. The subjects were 7 male Long-Evans rats, born and reared in the animal facilities at Southern Methodist University. All other subject and maintenance characteristics were similar to those described in the previous studies.

Apparatus. The eight-arm maze used in this study was similar to the maze used in the other studies, except that it was elevated only $35 \mathrm{~cm}$ from the floor and was located in a smaller room.

Procedure. After the animals had been accustomed to a waterdeprivation schedule in their home cages for about 2 weeks, a baseline phase of maze training began. The animals were individually placed on the maze and were allowed to choose eight arms per day. After 16 such baseline trials were conducted, each with water rewards at the end of each arm, the subjects had learned to select between seven and eight different arms each day.

The next phase was designed to condition an aversion to the taste of saccharin. At approximately $1000 \mathrm{~h}$, a drinking bottle filled with saccharin solution $(.15 \%, w / v)$ was placed on the home cage and the animal was allowed to drink for $10 \mathrm{~min}$. A lithium injection $(.15 \mathrm{M}, 20 \mathrm{cc} / \mathrm{kg}$ body weight, ip) was then administered to each subject. This saccharin-conditioning procedure was repeated every other day for a total of four conditioning trials. An intense aversion to saccharin was thus established. After the second conditioning trial, the subjects merely sniffed the saccharin solution and did not drink any of it.
The final testing phase began 2 days after the saccharin conditioning treatments in the home cage. During testing, the animals were once again placed on the maze and allowed to choose eight arms. The only difference between this phase and the baseline phase was that one of the arms contained a saccharin solution instead of water. This target location was determined randomly for each animal, with the restriction that the target not be the same for any two animals. As in baseline, the animals were administered one test trial per day. Fourteen tests were conducted over successive days.

\section{Results}

Group mean preferences for the target saccharin location over the final three blocks of baseline and the seven blocks of testing are plotted in Figure 5. The data reveal that preferences for the saccharin arm decreased steadily over repeated tests. Compared with the final three baseline blocks, preference for the target was significantly reduced by the last two blocks of testing $[t(6)=2.86$, $p<.05$ ]. Preference for the target on the initial test block (Block 4) was also significantly higher than that during the final two test blocks [smaller $t(6)=3.54, p<.05$ ]. This pattern reflects the decrease in arm preference developed during testing with the aversive saccharin.

Although the target arm in this study was different for each subject, this seems to have made little difference in the outcomes. Group mean preference scores dropped steadily during testing, as predicted. Although odor trails and other intramaze cues seem unimportant in guiding behavior on the maze in most cases (Olton \& Collison, 1979), it is worth noting that changes in behavior observed here are not controlled by these mechanisms.

It is also of interest to note that preference for an arm containing aversive saccharin declined even when this taste was rendered aversive in another location (i.e., the home cage). This indicates that taste-aversion conditioning need not be contingent on consumption of the toxic substance in the foraging location for preference reductions to take place in this location.

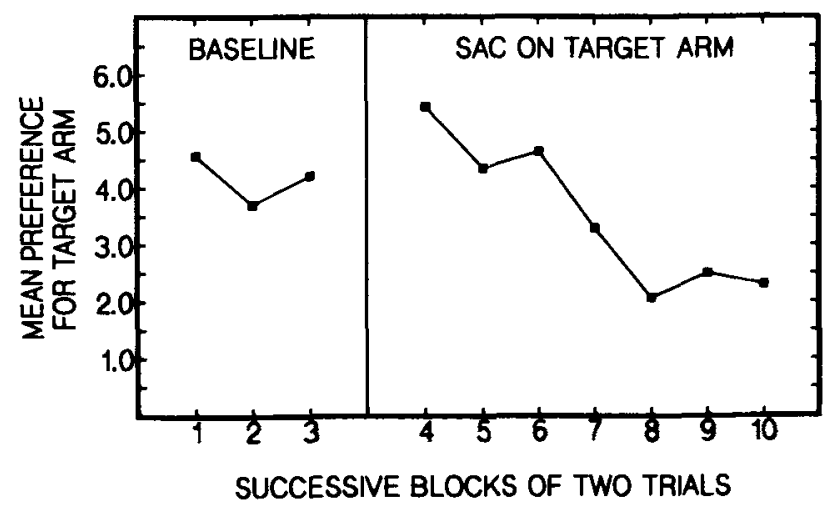

Figure 5. Mean preferences for the target arm during Experiment 3B. After the animals had learned to collect water rewards on the maze (baseline), they experienced saccharin-lithium pairings in the home cage environment. Finally, they were given a series of maze tests during which the aversive saccharin was located on a target arm of the maze. The target was different for each animal. 


\section{Discussion of Experiments $3 A$ and $3 B$}

The results of Experiments $3 \mathrm{~A}$ and $3 \mathrm{~B}$ demonstrate that arm preference can be reduced by tastes made aversive either contingent on exploration of the arm or in the home cage prior to testing. This avoidance of the target site might be mediated by one or more of several distinct processes. Animals' preferences for the saccharin arm may have decreased because lithium illness rendered the saccharin location aversive. One possible account is that the aversive taste conditioned the environmental stimuli through the process of second-order conditioning; the pairing of the aversive taste with specific arm stimuli each trial could cause these environmental stimuli to become aversive. In this context, Archer and Sjöden (1982) have demonstrated an important role for environmental stimuli in a variety of taste conditioning preparations, including second-order conditioning.

Alternatively, taste-mediated potentiation may have contributed to the decreased preference for the saccharin arm in these experiments. Recent reports have demonstrated that environmental, noningestional stimuli can be rendered aversive if a distinct taste is ingested in the presence of these stimuli and this encounter is followed by lithium-induced toxicosis (M. R. Best, Brown, \& Sowell, 1984). However, one requirement for producing this potentiation effect is the conditioning of the taste cues in the presence of the environmental stimuli (M. R. Best, Batson, Meachum, Brown, \& Ringer, 1985). This requirement was met in Experiment 3A, and taste-mediated environmental potentiation may have played a role in the arm preference declines observed. Indeed, this process has been shown to reduce instrumental performance in a straight alley (M. R. Best, Davis, Nash, \& Meachum, 1985). In this regard, however, the arm preference decline observed in Experiment $3 \mathrm{~B}$ is unlikely to be due to this potentiation process. The saccharin here was made aversive in the home cage, not in the maze.

It can also be argued that animals in the present study avoided the saccharin arm because there was no acceptable bait there. This interpretation is favored by Melcer and Timberlake (1985). Rats in that study were allowed to make repeated visits to all arms of a four-arm maze during a 20-min testing session. One group encountered one unbaited maze arm, and another group found a saccharin solution on one arm, after which it was injected with lithium. Both these groups later made fewer visits to the target (the location either unbaited or baited with saccharin) than did a control group. Moreover, saccharinconditioned animals visited the saccharin location no less often than other animals visited the unbaited arm. Melcer and Timberlake suggest that the saccharin-conditioned animals did not learn an aversion to the target environmental location, but rather avoided the saccharin arm for the same reason that the other animals avoided the unbaited arm: "because it did not contain an edible substance"' (p. 67).

If animals in the present study avoided the saccharin arm because it contained nothing edible, preferences for this arm should have decreased gradually as the animals learned there was nothing acceptable there. In fact, compared with the baseline behavior of the experimental group of Experiment 3A, there was a marked decrease in target preference on the first test trial following the initial conditioning trial $[t(3)=2.72, p<.05$, one-tailed]. Control animals showed no such significant difference $[t(3)$ $=1.07$, n.s.]. On the other hand, significant declines in preference for the saccharin location did not emerge until the ninth trial in Experiment 3B. Unlike the animals in Experiment 3A, the rats in Experiment 3B encountered, in the target location of the maze, saccharin that had been made aversive in the home cage. This indicates further that the processes mediating the arm-preference changes observed in Experiments $3 \mathrm{~A}$ and $3 \mathrm{~B}$ might be due to different processes.

\section{GENERAL DISCUSSION}

This report helps to document rats' responses to radialarm maze locations when reward quality is altered. Animals accustomed (during baseline) to drinking water in a specific location decreased their tendencies to visit that location when the reward was not present during subsequent trials (Experiment 1). Other animals increased their tendencies to visit a target spot when a highly palatable and highly caloric food was found there (Experiment 2). Finally, animals tended to decrease their visits to a target location that held an aversive saccharin solution (Experiments 3A and 3B). Optimal foraging theories (e.g., Kamil \& Sargent, 1981; Krebs \& McCleery, 1984; Pyke, Pulliam, \& Charnov, 1977) stress that behavior is modified according to cost-benefit functions. Behaviors that expend resources (in this situation, calories or time required to visit various maze locations) but which do not result in replenishment of resources (e.g., calories, water) should occur less often than behaviors that result in some definite reward or avoidance of aversive stimulation (e.g., illness). The results of the studies reported here seem to fit within such a framework.

Melcer and Timberlake (1985) have recently reported experiments that are similar to those reported here. Some of their subjects were tested on a four-arm radial maze with one location consistently unbaited. Other subjects were tested on the maze when an aversively conditioned saccharin solution was located on a specific maze arm. The present experiments generally replicate the results reported by these other authors. However, the present studies are different from those of Melcer and Timberlake in some important ways. First, Experiment 2 documents an increase in arm preference for a palatable and highly caloric bait. To our knowledge, no other studies have shown increases in willingness to visit a specific maze location. Second, the nature of a "trial" was different. Preferences in the present experiments were measured within trials of eight-arm choices each. In Melcer and Timberlake's studies, animals were allowed to visit all maze locations repeatedly during 20 -min test periods and 
the percentage of visits to the target, relative to visits to other arms, was recorded. This difference in dependentvariable computation is important since it might affect one's conclusions about changes in foraging behaviors. For example, preference for a given location within a series of eight-arm choices might be considered a more discrete measure of an animal's reactions to that location than Melcer and Timberlake's measure of percentage of visits to an arm over a period of time. On the other hand, the latter measure might more accurately represent the foraging behaviors of animals in the wild.

The present studies also raise questions about how animals learn to change their responses to specific environmental locations. Although these studies clearly show increases and decreases in willingness to visit various locations on the maze, they are not clear about the associative mechanisms underlying these changes. Did animals associate the quality of a bait with the location of the bait? If so, how is a flavor stimulus, for example, able to become associated with an environmental location? Answers to these and other questions would be most valuable in furthering our understanding of foraging behaviors.

Several investigations have recently shown that a distinctive flavor paired with poison may imbue aversive qualities to the place where the flavor was encountered. For example, M. R. Best et al. (1984) demonstrated that lithium conditioned not only an aversion to a saccharin solution but also to the box where the saccharin was consumed. Moreover, this "potentiation" of environmental conditioning is decreased when the aversive taste is extinguished in a separate context (M. R. Best et al., 1985). These results and those in the present series of experiments suggest that evolution has shaped animals not only to learn aversions to specific toxic flavors, but also to environments consistently containing those flavors.

\section{REFERENCES}

ARCher, T., \& SJöDEN, P. -O. (1982). Higher-order conditioning and sensory preconditioning of a taste aversion with an exteroceptive $\mathrm{CS}_{\mathbf{I}}$. Quarterly Journal of Experimental Psychology, 34B, 1-17.

Batson, J. D., \& Best, P. J. (1979). Drig-preexposure effects in flavoraversion learning: Associative interference by conditioned environmental stimuli. Journal of Experimental Psychology: Animal Behavior Processes, 5, 272-283.

Best, M. R., Batson, J. D., Meachum, C. L., Brown, E. R., \& RINGER, M. (1985). Characteristics of taste-mediated environmental potentiation in rats. Learning \& Motivation, 16, 190-209.

Best, M. R., Brown, E. R., \& Sowell, M. K. (1984). Taste-mediated potentiation of noningestional stimuli in rats. Learning \& Motivation, 15, 244-258
Best, M. R., Davis, S. F., NASH, S. M., \& Meachum, C. L. (1985, August). Taste aversion learning: Locus of conditioning affects straight runway performance. Poster presented at the meeting of the American Psychological Association, Los Angeles, CA.

Best, P. J., Best, M. R., \&engGeler, S. (1977). The contribution of environmental non-ingestive cues in conditioning with aversive internal consequences. In L. M. Barker, M. R. Best, \& M. Domjan (Eds.), Leaming mechanisms in food selection (pp. 371-393). Waco, TX: Baylor University Press.

Bolles, R. C., Hayward, L., \& Crandall, C. (1981). Conditioned taste preferences based on caloric density. Journal of Experimental Psychology: Animal Behavior Processes, 7, 59-69.

EINON, D. (1980). Spatial memory and response strategies in rats: Age, sex and rearing differences in performance. Quarterly Journal of Experimental Psychology, 32, 473-489.

FLAHERTY, C. F. (1982). Incentive contrast: A review of behavioral changes following shifts in reward. Animal Learning \& Behavior, 10 , 409-440.

Foreman, N. (1985). Algorithmic responding on the radial maze in rats does not always imply absence of spatial encoding. Quarterly Journal of Experimental Psychology, 37B, 333-358.

KAmIL, A. C., \& SARgent, T. E. (Eds.) (1981). Foraging behavior: Ecological, ethological and psychological approaches. New York: Garland.

KIRK, R. E. (1968). Experimental design: Procedures for the behavioral sciences. Belmont, CA: Brooks/Cole.

KREBS, J. R., \& MCCLEERY, R. H. (1984). Optimization in behavioural ecology. In J. R. Krebs \& N. B. Davies (Eds.), Behavioural ecology: An evolutionary approach (2nd ed., pp 91-121). Sunderland, MA: Sinauer.

Mehiel, R., Bolles, R. C. (1984). Learned flavor preferences based on caloric outcome. Animal Learning \& Behavior, 12, 421-427.

Melcer, T., \& Timberlake, W. (1985). Poison avoidance and patch (location) selection in rats. Animal Leaming \& Behavior, 13, 60-68.

Olton, D. S. (1978). Characteristics of spatial memory. In S. H. Hulse, H. Fowler, \& W. K. Honig (Eds.), Cognitive processes in animal behavior (pp. 341-373). Hillsdale, NJ: Erlbaum.

Olton, D. S., \& Coluson, C. (1979). Intramaze cues and "odor trails" fail to direct choice behavior on an elevated maze. Animal Learning \& Behavior, 7, 221-223.

Olton, D. S., SAMUELSON, R. J. (1976). Remembrance of places passed: Spatial memory in rats. Journal of Experimental Psychology: Animal Behavior Processes, 2, 97-116.

Pyke, G. H., Pulliam, H. R., \& Charnov, E. L. (1977). Optimal foraging: A selective review of theory and tests. Quarterly Review of Biology, 52, 137-154.

SPENCE, K. W., \& LiPPITT, R. D. (1946). An experimental test of the sign-gestalt theory of trial and error learning. Journal of Experimental Psychology, 36, 491-502.

Yoerg, S. I., KAMIL, A. C. (1982). Response strategies in the radial arm maze: Running around in circles. Animal Learning \& Behavior, $10,530-534$.

(Manuscript received January 6, 1986; revision accepted for publication June $30,1986$. ) 